\section{Protective Effect of Anthocyanidins on Astrocytes and Apoptosis Induced by Oxidative Damage}

\section{Ana López, Olga Palomino, Teresa Ortega, Emilia Carretero}

Pharmacology Department, Faculty of Pharmacy, Universidad Complutense de Madrid, Ciudad Universitaria s/n, Madrid, Spain

\section{Abstract \\ $\nabla$}

As a result of the longer life expectancy in the human population, neurodegenerative diseases are increasing, so finding effective treatments together with prevention strategies are urgently needed. Anthocyanins are polyphenolic compounds from vegetal species that have shown strong antioxidant and anti-inflammatory activities in various experimental models. The effect of the anthocyanidins cyanidin, malvidin, and pelargonidin at different concentrations ( 5 to $100 \mu \mathrm{M}$ ) on experimental models of cell oxidation and apoptosis was studied and compared with the positive control Trolox. The human astrocyte glioblastoma, cellular line U373, was used for a cellular injury model using Fenton's reagent $\left(0.5 \mathrm{mM} \mathrm{FeSO}_{4} / 1 \mathrm{mM} \mathrm{H}_{2} \mathrm{O}_{2}\right)$ and to measure the lactate dehydrogenase enzyme activities and lipid peroxidation level (thiobarbituric acid reactive substances) by fluorimetric analysis and malondialdehyde release by HPLC. Also, caspase 3, 8, and 9 activities were measured by fluorimetric analysis. The results show that the studied anthocyanidins are able to revert the induced apoptosis related to caspase activation and lipid peroxidation. All the assayed anthocyanidins significantly decreased the activation of caspases 8 and 3, although malvidin and cyanidin increased the activity of caspase 9 when compared to non-treated cells.

\section{Key words}

antioxidant $\cdot$ neuroprotection · anthocyanidins $\cdot$ cyanidin $\cdot$ pelargonidin $\cdot$ malvidin $\cdot$ oxidative stress

Neurodegenerative diseases are known to be related to oxidative stress which is due to an imbalance between the production of reactive oxygen species (ROS) and the ability of intrinsic antioxidant systems, both enzymatic and non-enzymatic, to scavenge these radicals [1]. Oxidation of macromolecules such as proteins, lipids and DNA may lead to cell degeneration and death due to an increase in the release of apoptotic-inducing factors. Thus, different strategies have been proposed for the prevention and treatment of ROS-mediated diseases, with special emphasis on antioxidant therapy [2-4].

Anthocyanidins are polyphenols with a strong scavenging activity, which is related to their phenolic structure characterized by $B$ ring hydroxyl groups and a conjugated double bond system. Orally administered anthocyanins have been proven to suppress stress-induced cerebral oxidative damage [5], reduce lipid peroxidation, increase glutathione levels and antioxidant enzymes activity, and improve spatial memory in the hippocampus of the adult rat [6].

In this study, we analyzed the antioxidant activity of the anthocyanidins cyanidin, malvidin, and pelargonidin at different concentrations on the human astrocyte glioblastoma, which is known as a useful model for the study of astrocyte functions under both physiological and pathological conditions. Then, the potential protective effect of these compounds against an oxidative injury chemically induced by Fenton's reagent, which induces a double-strand DNA breaking, was assessed [7]. For this purpose, lactate dehydrogenase (LDH) activity and the lipid peroxidation level through thiobarbituric acid reactive substances (TBARS) and malondialdehyde (MDA) levels were evaluated. Finally, apoptotic caspases 3,8 , and 9 were tested to determine whether anthocyanidins may influence cell apoptosis. Trolox (6hydroxy-2,5,7,8-tetramethylchroman-2-carboxylic acid) is a water-soluble analogue of vitamin $\mathrm{E}$ with strong antioxidant capacity that is widely used as a positive control in several tests conducted to assay the antioxidant ability of new compounds. It is able to diminish ROS production, block the loss of MMP, prevent cytotoxicity in human cancer cell lines, and rescue cells from apoptotic death $[8,9]$. Trolox was chosen as a positive control in all the assays conducted in this work.

\section{Results and Discussion \\ $\nabla$}

The direct effect of the anthocyanidins on astrocyte viability (LDH) showed a statistically significant lower LDH release than the toxic control (Fenton's reagent), similar to the positive control ( $0.5 \mathrm{mM}$ Trolox) but higher than untreated cells, except for $25 \mu \mathrm{M}$ cyanidin ( Fig. 1 A). Their protective effect was assessed by a decrease in LDH release, although with a different profile. For cyanidin, the highest tested concentration showed the highest LDH value, while the mean concentration $(25 \mu \mathrm{M})$ showed the lowest LDH value. Pelargonidin induced a dose-dependent production of $\mathrm{LDH}$, while the inverse profile was observed for malvidin, as lower LDH levels were detected for the higher concentrations. These results were similar than those observed for the positive control ( $\odot$ Fig. 1 B).

MDA, the main compound resulting from lipid peroxidation, was evaluated in the presence or absence of oxidative stress conditions. In the absence of an oxidative stimulus, no statistically significant differences were found between anthocyanidin concentrations, Trolox treatment, and control cells on TBARS levels, except for $5 \mu \mathrm{M}$ cyanidin and $25 \mu \mathrm{M}$ malvidin ( $\odot$ Fig. $2 \mathrm{~A}$ ). Under oxidative stress injury, a protective effect was shown through a reduction in these values ( $\bullet$ Fig. 2 B).

Under normal conditions, every anthocyanidin induced statistically significant lower MDA levels than the toxic alone, and similar to that of the control and Trolox groups ( Fig. 3A), except for pelargonidin at the highest concentration. When the cells were previously treated with anthocyanidins, the lipid peroxidation damage induced by Fenton's reagent was significantly decreased, even more than the positive control, Trolox ( $\odot$ Fig. 3B).

A direct effect of cyanidin, malvidin, and pelargonidin on caspase activities showed no significant differences for caspases 3 and 8 . Significant differences were found between the control and malvidin-treated cells for caspase 9 activity; also, $25 \mu \mathrm{M}$ cyanidin showed a higher caspase 9 activity than untreated cells ( Fig. 4A). When testing the protective effect on a cellular injury model induced by $500 \mu \mathrm{M} \mathrm{H}_{2} \mathrm{O}_{2}$ ( $\odot$ Fig. 4B), every tested compound reduced caspase 3 and 8 activities, while caspase 9 was not activated by the toxic treatment.

This study demonstrates that cyanidin, malvidin, and pelargonidin are capable of protecting human astrocytes against oxidative injury mainly influencing caspase activity. The potential preventive effect of the anthocyanidins when an injured cell model was 


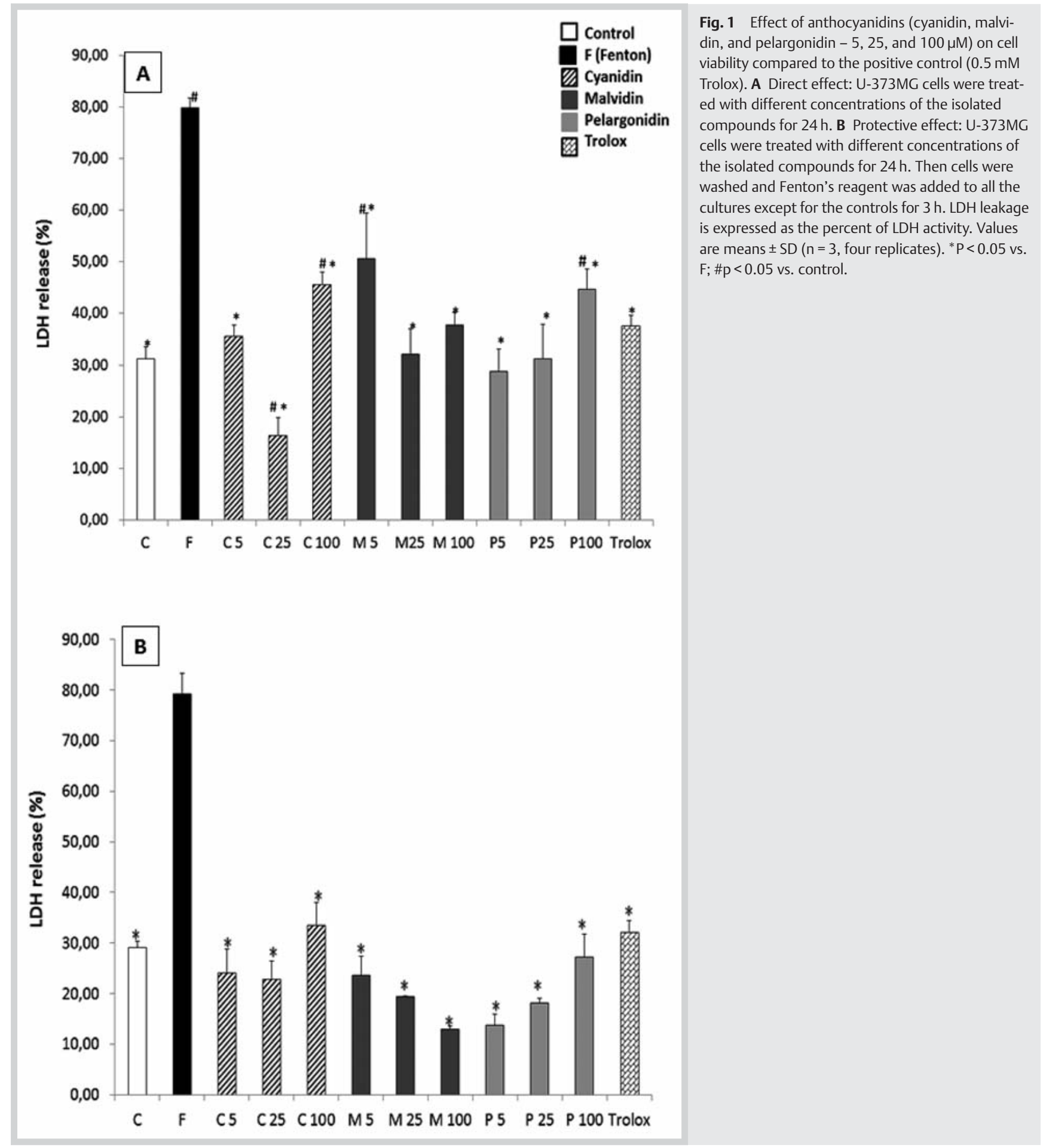

implemented proved their ability to reduce LDH release to culture media up to values close to untreated cells, but with a different behavior, probably due to the different chemical structure of these compounds. Under oxidative stress conditions, polyunsaturated fatty acids of cell membranes may break down to different secondary products such as aldehydes with MDA being one of the main products derived from lipid peroxidation, which is widely used as an index of peroxidation in biological sciences $[10,11]$. In this study, an HPLC method was applied to quantify the peroxidation metabolite (data not shown). Results proved the protective effect from anthocyanidins, but with a different profile. These differences may be related to the structure-activity relationship within anthocyanidins, as anthocyanidin trihydroxilated derivates are more effective in neutralizing ROS than monohydroxilated ones (pelargonidin), which in turn are more efficient in inhibiting proteasome activity (which could also inhibit the nuclear factor kappa-light-chain-enhancer of activated $\mathrm{B}$ cells-NF- $k$ B pathway) [12].

These results are in accordance with previous studies that proved a similar effect of cyanidin-3-glucoside over endothelial cells 


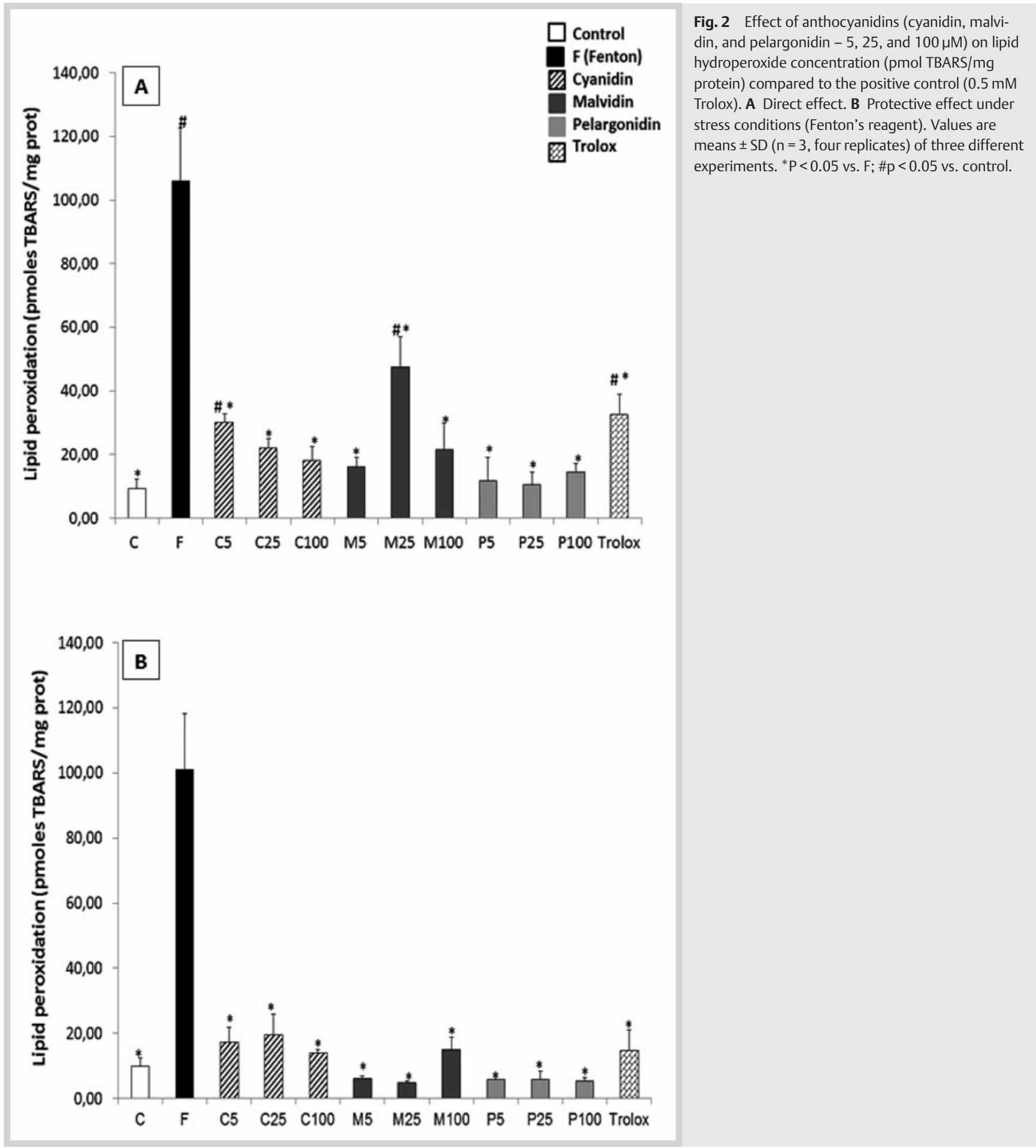

[13], as well as other anthocyanidin-rich products such as beans, which significantly reduced free radical time-dependent generation and exerted antioxidant activity [14].

In this work, the effect of anthocyanidins showed no significant differences between treatments on caspase 3 (executioner) activity and untreated cells; neither were differences found for caspase 8 (initiator) activity. However, every malvidin concentration and $25 \mu \mathrm{M}$ cyanidin improved caspase 9 activity (initiator). The study on the protective effect after oxidative injury showed a decrease in caspase 3 and 8 activities, with no statistically significant differences between the compounds and concentrations. In view of the results, and taking into account the previous studies in this field, it is proposed that the protective effect of anthocyanidins should be initiated via an extrinsic pathway and not an intrinsic one [6,15-18].

The results shown in this work support previous data on the strong antioxidant effect of anthocyanidins in several experimental models. The inhibitory effect on lipid peroxidation, together with direct antioxidant and antiapoptotic effects under oxidative stress injury on astrocytes was assessed. These results may contribute to the knowledge of the molecular mechanism involved in such an antioxidant effect, although more in-depth 


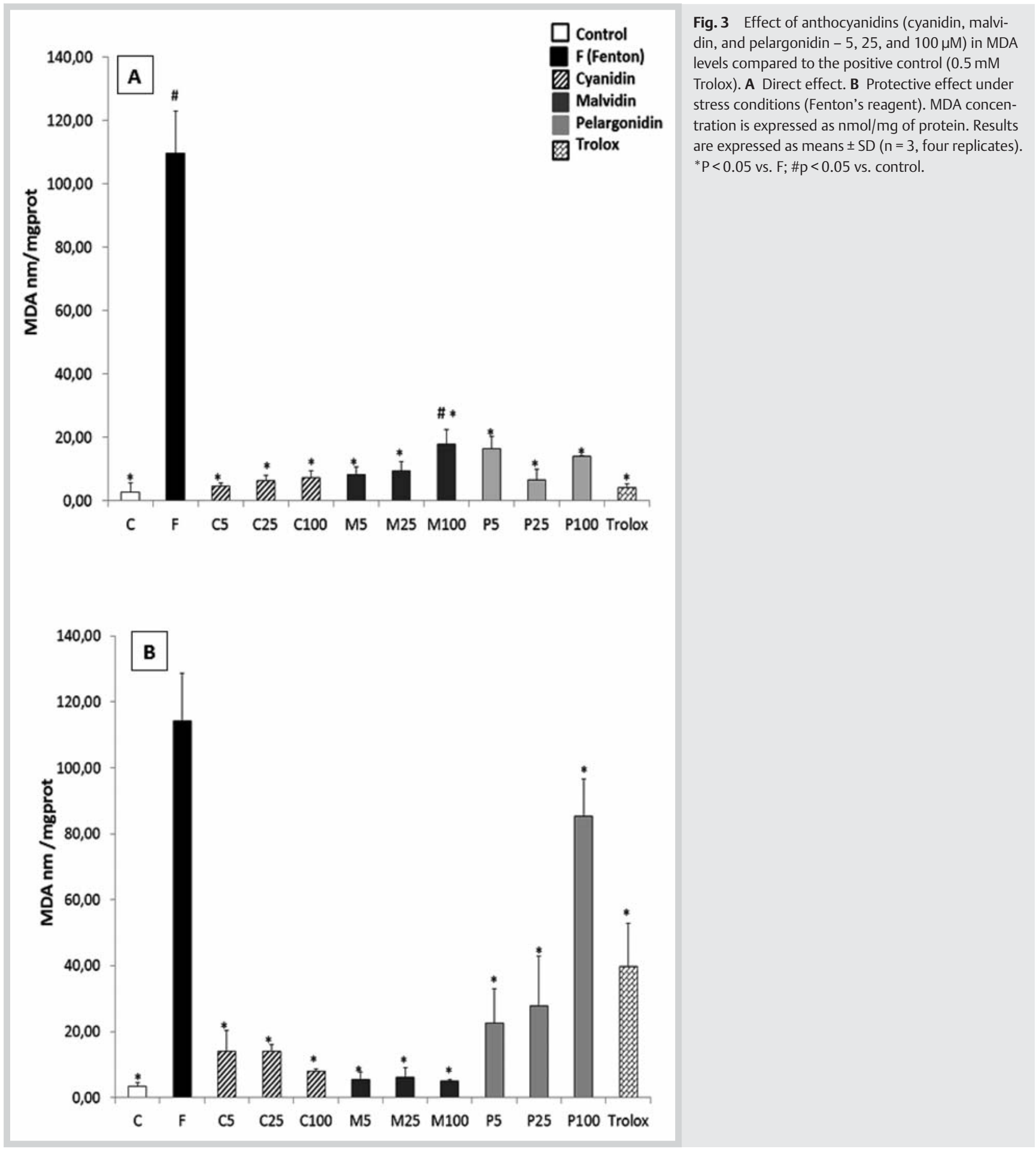

research is needed to evaluate their possible usefulness as natural protection against those diseases in which oxidative stress plays a crucial role.

\section{Materials and Methods}

$\nabla$

\section{Reagents}

Cyanidin, malvidin, and pelargonidin were purchased from Extrasynthese (France) with a purity $\geq 96 \%$. Gentamicin, penicillin, and streptomycin were purchased from Sigma Chemical (Spain).
Trolox (97\% purity) was purchased from Sigma-Aldrich (Spain). Fenton reagent $\left(\mathrm{FeSO}_{4} / \mathrm{H}_{2} \mathrm{O}_{2}\right)$ and other reagents were of analytical or chromatographic quality.

\section{Cell culture}

The human astrocytoma U373 MG line was obtained from the Cell Culture and Biological Resources Unit at Alcala de Henares University (Madrid, Spain). Cells were grown in a humidified incubator at $5 \% \mathrm{CO}_{2}$ and $95 \%$ air at $37{ }^{\circ} \mathrm{C}$ in Dulbecco's modified Eagle's medium (DMEM) piruvate free, from Invitrogen (Madrid, 


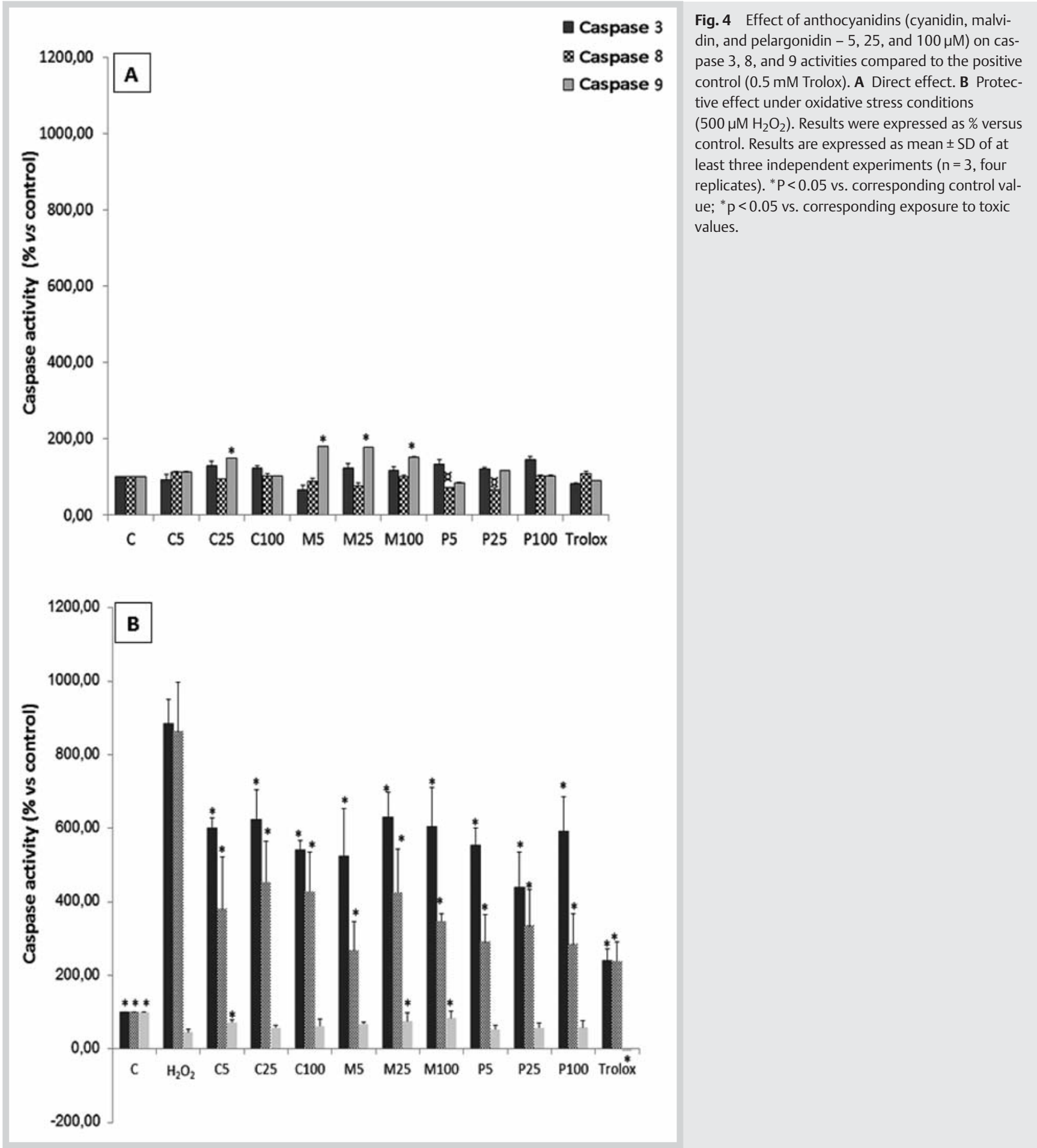

Spain), supplemented with $10 \%$ fetal bovine serum (FBS) (Biowhitaker) and $50 \mathrm{mg} / \mathrm{l}$ of each one of the following antibiotics: gentamicin, penicillin, and streptomycin.

\section{Cell treatment}

Three concentrations of cyanidin, malvidin, and pelargonidin (5, 25 , and $100 \mu \mathrm{M}$ ) were dissolved in DMEM and added to the cell plates for $24 \mathrm{~h}$. In order to evaluate the protective effect against an oxidative insult, the culture medium was removed and the cells were treated with Fenton reagent $\left(0.5 \mathrm{mM} \mathrm{FeSO}_{4} / 1 \mathrm{mM}\right.$ $\mathrm{H}_{2} \mathrm{O}_{2}$ ) for $45 \mathrm{~min}$.
For all of the experiments, every sample was analyzed in triplicate, with four plates for each condition.

\section{Lactate dehydrogenase activity assay}

Cells $\left(2 \times 10^{5}\right)$ were seeded in 24 -well plates and treated as described in the previous section. Then, $100 \mu \mathrm{L}$ of each plate were transferred into 96-multiwell plates, while the remaining cells were treated with the lysis buffer (Triton X-100) and transferred into Eppendorf tubes. Sodium pyruvate $80 \mathrm{mM}$ and NADH ( $2 \mathrm{mg}$ / $\mathrm{ml}$ ) were added just before reading both samples (culture medium and lysate) [19]. 
The LDH leakage was measured using a Fluostar optima fluorimeter ( $\lambda 340 \mathrm{~nm}, 37^{\circ} \mathrm{C}$ for $10 \mathrm{~min}$ ) and expressed as a percentage from the ratio between the LDH activity in the culture medium and that of the whole cell content [9].

\section{Lipid peroxidation}

Quantification of MDA as the major end product of lipid peroxidation was carried on by chromatographic evaluation and the TBARS fluorimetric assay.

HPLC [20]: Briefly, samples were filtered through one cellulose acetate filter $(0.22 \mu \mathrm{m})$ and injected on an Agilent 1100 series HPLC-DAD chromatograph with an isocratic elution of methanol : water $(50: 50 \mathrm{~V} / \mathrm{V})$ for $20 \mathrm{~min}$. The flow rate was $0.5 \mathrm{~mL} / \mathrm{min}$ and detection was set at 268 and $532 \mathrm{~nm}$ within a photodiode array detector. MDA values are expressed as nanomoles of MDA per milligram of protein.

TBARS Fluorometric Assay [21]: Samples were kept at $90^{\circ} \mathrm{C}$ for $45 \mathrm{~min}$ at a low $\mathrm{pH}$. Then, thiobarbituric acid was added to yield a fluorescent red chromogen that was read at $530 \mathrm{~nm}$. The results are expressed as a percentage of TBARS, as referred to the toxic value (100\%).

\section{Caspase 3, 8, and 9 determinations}

The fluorogenic caspases 3, 8, and 9 were used for a fluorimetric assay. After the hydrolytic cleavage of the coumarin derivative by the cytosolic caspases, the fluorescent product was determined using a multiwell fluorescence reader (Bio-Tek Instruments). Fluorescence was measured at $360 \mathrm{~nm}$ excitation and $480 \mathrm{~nm}$ emission for caspase 3; emission was set at $528 \mathrm{~nm}$ for caspases 8 and 9.

\section{Statistical analysis}

Statgraphics Centurion 16.1.15 (XV) was used. One-way analysis of variance (ANOVA) followed by Fishers least significant difference (LSD) test was applied to obtain the differences between samples. $\mathrm{P}<0.05$ was considered statistically significant.

\section{Conflict of Interest}

$\nabla$

The authors have no conflicts of interest to declare.

\section{References}

1 Inglese $M$, Petracca M. Imaging multiple sclerosis and other neurodegenerative diseases. Prion 2013; 7: 47-54

2 Connolly PF, Jäger R, Fearnhead HO. New roles for old enzymes: killer caspases as the engine of cell behavior changes. Front Physiol 2014; 5 : 149

3 Emerit J, Edeas M, Bricaire F. Neurodegenerative diseases and oxidative stress. Biomed Pharmacother 2004; 58: 39-46

4 Valko M, Rhodes CJ, Moncol J, Izakovic M, Mazur M. Free radicals, metals and antioxidants in oxidative stress-induced cancer. Chem Biol Interact 2006; 160: $1-40$

5 Roghani M, Niknam A, Jalali-Nadoushan MR, Kiasalari Z, Khalili M, Baluchnejadmojarad T. Oral pelargonidin exerts dose-dependent neuroprotection in 6-hydroxydopamine rat model of hemi-parkinsonism. Brain Res Bull 2010; 82: 279-283

6 Shah SA, Yoon GH, Kim MO. Protection of the developing brain with anthocyanins against ethanol-induced oxidative stress and neurodegeneration. Mol Neurobiol, advance online publication July 2014; DOI: 10.1007/s12035-014-8805-7

7 Moriwaki H, Osborne MR, Phillips DH. Effects of mixing metal ions on oxidative DNA damage mediated by a Fenton-type reduction. Toxicol In Vitro 2008; 22: 36-44

8 Kello M, Drutovic D, Chripkova M, Pilatova M, Budovska M, Kulikova L, Urdzik P, Mojzis J. ROS-dependent antiproliferative effect of brassinin derivative homobrassinin in human colorectal cancer Caco2 cells. Molecules 2014; 19: 10877-10897

9 Schoeneberger H, Belz K, Schenk B, Fulda S. Impairment of antioxidant defense via glutathione depletion sensitizes acute lymphoblastic leukemia cells for Smac mimetic-induced cell death. Oncogene, advance online publication Nov 2014; DOI: 10.1038/onc.2014.338

10 Li P, Ding G, Deng Y, Punyapitak D, Li D, Cao Y. Determination of malondialdehyde in biological fluids by high-performance liquid chromatography using rhodamine $\mathrm{B}$ hydrazide as the derivatization reagent. Free Radic Biol Med 2013; 65: 224-231

11 Martín MA, Ramos S, Mateos R, Granado AB, Izquierdo-Pulido M, Bravo L, Goya L. Protection of human HepG2 cells against oxidative stress by cocoa phenolic extract. J Agric Food Chem 2008; 56: 7765-7772

12 Pascual-Teresa S. Molecular mechanisms involved in the cardiovascular and neuroprotective effects of anthocyanins. Arch Biochem Biophys 2014; 559: 68-74

13 Speciale A, Canali R, Chirafisi J, Saija A, Virgili F, Cimino F. Cyanidin-3-Oglucoside protection against TNF- $\alpha$-induced endothelial dysfunction: involvement of nuclear factor- $k$ B signaling. J Agric Food Chem 2010; 58: $12048-12054$

14 López A, El-Naggar T, Dueñas M, Ortega T, Estrella I, Hernández T, Gómez-Serranillos MP, Palomino OM, Carretero ME. Effect of cooking and germination on phenolic composition and biological properties of dark beans (Phaseolus vulgaris L.). Food Chem 2013; 138: 547-555

15 Kim HG, Ju MS, Shim JS, Kim MC, Lee SH, Huh Y, Kim SY, Oh MS. Mulberry fruit protects dopaminergic neurons in toxin-induced Parkinson's disease models. Br J Nutr 2010; 104: 8-16

16 Li Q Li C, Mahtani HK, Du J, Patel AR, Lancaster JR jr. Nitrosothiol formation and protection against Fenton chemistry by nitric oxide-induced dinitrosyl iron complex formation from anoxia-initiated cellular chelatable iron increase. J Biol Chem 2014; 289: 19917-19927

17 Jang H, Bae WJ, Kim SJ, Yuk SM, Han DS, Ha US, Hwang SY, Yoon SH, Wang $Z$, Kim SW. The effect of anthocyanin on the prostate in an andropause animal model: rapid prostatic cell death by apoptosis is partially prevented by anthocyanin supplementation. World J Mens Health 2013; 31: 239-246

18 Ullah I, Park HY, Kim MO. Anthocyanins protect against kainic acid-induced excitotoxicity and apoptosis via ROS-activated AMPK pathway in hippocampal neurons. CNS Neurosci Ther 2014; 20: 327-338

19 Koh JY, Choi DW. Quantitative determination of glutamate mediated cortical neuronal injury in cell culture by lactate dehydrogenase efflux assay. J Neurosci Methods 1987; 20: 83-90

20 Grotto D, Santa María LD, Boeira S, Valentini J, Charão MF, Moro AM, Nascimento PC, Pomblum VJ, García SC. Rapid quantification of malondialdehyde in plasma by high performance liquid chromatographyvisible detection. J Pharm Biomed Anal 2007; 43: 619-624

21 Schmidt M, Dringen R. Zinc prevents the copper-induced damage of cultured astrocytes. Neurochem Int 2010; 57: 314-322

received January 19, 2015

revised March 18, 2015

accepted March 20, 2015

Bibliography

Dol http://dx.doi.org/10.1055/s-0035-1545938

Planta Med Lett 2015; 2: e19-e24

(c) Georg Thieme Verlag KG Stuttgart · New York .

ISSN 2199-157X

\section{Correspondence}

\section{Univ. Prof. Dr. Teresa Ortega}

Faculty of Pharmacy

Universidad Complutense de Madrid

Ciudad Universitaria s/n

Department of Pharmacology

Plaza de Ramón y Cajal s/n

28040 Madrid

Spain

Phone: + 34913941871

Fax: +34913941726

tortega@farm.ucm.es 